PROCEEDINGS OF THE

AMERICAN MATHEMATICAL SOCIETY

Volume 130, Number 6, Pages 1611-1617

S 0002-9939(01)06246-3

Article electronically published on November 15, 2001

\title{
DIRECT SUMS OF LOCAL TORSION-FREE ABELIAN GROUPS
}

\author{
DAVID M. ARNOLD \\ (Communicated by Stephen D. Smith)
}

\begin{abstract}
The category of local torsion-free abelian groups of finite rank is known to have the cancellation and $n$-th root properties but not the KrullSchmidt property. It is shown that 10 is the least rank of a local torsionfree abelian group with two non-equivalent direct sum decompositions into indecomposable summands. This answers a question posed by M.C.R. Butler in the 1960's.
\end{abstract}

\section{INTRODUCTION}

Let $T F$ denote the category of local torsion-free abelian groups of finite rank, where an abelian group $G$ is local if there is a fixed prime $p$ with $q G=G$ for each prime $q \neq p$. Each $M$ in $T F$ has the cancellation property (if $M \oplus N$ is isomorphic to $M \oplus K$ in $T F$, then $N$ is isomorphic to $K$ ), and the $n$-th root property (if the direct sum $M^{n}$ of $n$ copies of $M$ is isomorphic to $N^{n}$ for some $N$ in $T F$, then $M$ is isomorphic to $N$ ) Lady 75. An $M$ in TF is a Krull-Schmidt group if any two direct sum decompositions of $M$ into indecomposable summands are equivalent, i.e. unique up to isomorphism and order of summands.

M.C.R. Butler, in an unpublished note dating from the 1960's, constructed an example of a local torsion-free abelian group of rank 16 that is not a Krull-Schmidt group (see Arnold 82]) and asked for the smallest such rank. An example of a rank-10 local torsion-free abelian group that is not a Krull-Schmidt group is given in Arnold 01.

This paper is devoted to showing that 10 is the minimum such rank, i.e. if $M \in T F$ with rank $M \leq 9$, then $M$ is a Krull-Schmidt group. Many arguments in this paper carry over directly to torsion-free modules of finite rank over valuation domains, keeping in mind that the existence and minimal rank of a non-Krull-Schmidt module depends on the structure of the valuation domain; see Goldsmith May 99. and references.

The quasi-isomorphism category $T F_{\mathbb{Q}}$ of $T F$ is an additive category with objects those of $T F$ but with morphism sets $\mathbb{Q} \otimes \operatorname{Hom}(M, N)$ for $M, N \in T F$ and $\mathbb{Q}$ the rational numbers. The category $T F_{\mathbb{Q}}$ is a Krull-Schmidt category in that each object in $T F_{\mathbb{Q}}$ can be written uniquely, up to isomorphism in $T F_{\mathbb{Q}}$ and order, as a finite direct sum of indecomposable objects in $T F_{\mathbb{Q}}$; see Walker 64. This is because

Received by the editors October 4, 2000 and, in revised form, January 8, 2001.

2000 Mathematics Subject Classification. Primary 20K15, $20 \mathrm{~K} 25$.

Key words and phrases. Krull-Schmidt groups, direct sum decompositions, local torsion-free abelian groups.

This research was supported, in part, by the Baylor University Summer Sabbatical Program. 
an indecomposable object $M$ in $T F_{\mathbb{Q}}$ has a local endomorphism ring $\mathbb{Q}$ End $M$ in $T F_{\mathbb{Q}}$. Indecomposable objects in $T F_{\mathbb{Q}}$ are called strongly indecomposable groups, isomorphism in $T F_{\mathbb{Q}}$ is called quasi-isomorphism, and summands of groups in $T F_{\mathbb{Q}}$ are called quasi-summands.

Each $M \in T F$ is a torsion-free $\mathbb{Z}_{(p)}$-module, where $\mathbb{Z}_{(p)}$ is the localization of the integers at the prime $p$. The rank of $M$ as a group is equal to the rank of $M$ as

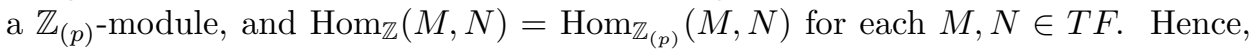
$\operatorname{rank} M=1$ if and only if $M$ is isomorphic to either $\mathbb{Z}_{(p)}$ or $\mathbb{Q}$. Define $p$-rank $M$ to be the $\mathbb{Z} / p \mathbb{Z}$-dimension of $M / p M$, a finite dimensional $\mathbb{Z} / p \mathbb{Z}$-vector space. Notice that $p$-rank $M \leq \operatorname{rank} M, M$ is divisible if and only if $p$-rank $M=0$, and $M$ is isomorphic to a free $\mathbb{Z}_{(p)}$-module if and only if $p$-rank $M=\operatorname{rank} M$. Moreover, if $N$ is a $\mathbb{Z}_{(p)}$-submodule of $M$, then the $p$-rank of the pure submodule of $M$ generated by $N$ is less than or equal to the $p$-rank of $N$ and if $M$ is quasi-isomorphic to $N$ $\oplus K$, then $p$-rank $M=p$-rank $N+p$-rank $K$ Arnold 72. If $M$ is reduced (no proper divisible subgroups), then $M$ is isomorphic to a pure subgroup of $M^{*}$, the completion of $M$ in the $p$-adic topology. Moreover, $M^{*}$ is a free $\mathbb{Z}^{*}$-module with rank equal to the $p$-rank of $M$, where $\mathbb{Z}^{*}$ is the $p$-adic completion of $\mathbb{Z}_{(p)}$. Each endomorphism of $M$ lifts to a unique $\mathbb{Z}^{*}$-endomorphism of $M^{*}$, whence End $M$ is a pure subring of $\operatorname{End}_{\mathbb{Z}^{*}} M^{*}$.

\section{UNIQUENESS OF DIRECT SUMS}

A group $M \in T F$ has the one-sided UDS property if whenever $M \oplus N$ is isomorphic to $K_{1} \oplus \ldots \oplus K_{n} \in T F$ with $M$ quasi-isomorphic to each $K_{j}$, then $M$ is isomorphic to some $K_{j}$. The group $M$ has the UDS property if whenever $N_{1} \oplus \ldots$ $\oplus N_{m}$ is isomorphic to $K_{1} \oplus \ldots \oplus K_{n} \in T F$ with $M$ quasi-isomorphic to each $N_{i}$ and $K_{j}$, then $m=n$ and there is a relabelling of indices with each $N_{i}$ isomorphic to $K_{i}$.

Given a strongly indecomposable $M$ in $T F$, there is a faithful $G_{M} \in T F$ quasiisomorphic to $M$ such that End $G_{M} / N$ End $G_{M}$ is a maximal order in the division algebra $\mathbb{Q E n d} M / J \mathbb{Q E n d} M$, where $J \mathbb{Q E n d} M$ is the Jacobson radical of the finite dimensional $\mathbb{Q}$-algebra $\mathbb{Q E n d} M, N$ End $G_{M}=$ End $G_{M} \cap J \mathbb{Q E n d} M$ is a nilpotent ideal of End $M$, and $G_{M}$ is faithful if $I G_{M} \neq G_{M}$ for each maximal right ideal $I$ of End $G_{M}$ [Arnold 01. A maximal right ideal $J$ of End $G_{M} / p$ End $G_{M}$ has the unique maximal condition if whenever $I$ is a non-zero right ideal and $J$ is a unique maximal right ideal of End $G_{M} / p$ End $G_{M}$ containing $I$, then $I=J$.

The first lemma is the local version of [Arnold 01, Theorem 1.5].

Lemma 1. The following statements are equivalent for a strongly indecomposable $N$ in $T F$, where $G_{N}$ is as defined above:

(i) $N$ has the UDS property.

(ii) Each group in TF quasi-isomorphic to $N$ has the one-sided UDS property.

(iii) Either End $G_{N}$ is a local ring or else End $G_{N}$ has exactly two maximal right ideals $M_{1}$ and $M_{2}$ such that $M_{1}$ is a principal right ideal of End $G_{N}, G_{N} / M_{1} G_{N} \cong$ $\mathbb{Z} / p \mathbb{Z}$, and $M_{1} / p$ End $G_{N}$ has the unique maximal condition in End $G_{N} / p$ End $G_{N}$.

Following are non-trivial examples of groups in $T F$ with the UDS property.

Example 1. If $N \in \mathrm{TF}$ is strongly indecomposable with $p$-rank $N \leq 2$, then $N$ has the (one-sided) UDS property. 
Proof. It suffices to confirm the conditions of Lemma 1(iii). If $p$-rank $N \leq 1$, then $p$-rank $G_{N} \leq 1$, since $G_{N}$ is quasi-isomorphic to $N$. Thus, either $p$-rank $G_{N}=0$ and $G_{N} \cong \mathbb{Q}$ or else $p$-rank $G_{N}=1, G_{N}^{*} \cong \mathbb{Z}^{*}$, and End $G_{N}$ is isomorphic to a pure subring of $\mathbb{Z}^{*} \cong \operatorname{End}_{\mathbb{Z}^{*}} \mathbb{Z}^{*}$. In either case, End $G_{N}$ is a local ring, as desired.

Now assume that $p$-rank $N=p$-rank $G_{N}=2$ and End $G_{N}$ is not a local ring. Let $M_{1}, \ldots, M_{n}$ be distinct maximal right ideals of End $G_{N}$ with $n \geq 2$. Then $G_{N} /\left(M_{1} \cap \ldots \cap M_{n}\right) G_{N} \cong G_{N} / M_{1} G_{N} \oplus \ldots \oplus G_{N} / M_{n} G_{N}$ and $p$ End $G_{N} \subseteq J$ End $G_{N} \subseteq M_{1} \cap \ldots \cap M_{n}$. Since $p$-rank $\mathrm{G}_{N}=2$ and $G_{N}$ is faithful, it follows that $n=2, p$ End $G_{N}=M_{1} \cap M_{2}=J$ End $G_{N}$, each $G_{N} / M_{i} G_{N} \cong \mathbb{Z} / p \mathbb{Z}$, and each $M_{i} / p$ End $G_{N}$ has the unique maximal condition. Finally, each $M_{i}$ is principal as an application of Nakayama's Lemma, because $p$ End $G_{N}=J$ End $G_{N}$ and End $G_{N} / p$ End $G_{N}$ is finite.

The next lemma is used for an induction step in the proof of the main theorem.

Lemma 2. Assume that $M=N \oplus N^{\prime}=K_{1} \oplus \ldots \oplus K_{n} \in T F$. There are subgroups $K_{i}^{\prime}$ of $K_{i}$ with $N \oplus N^{\prime}=N \oplus K_{1}^{\prime} \oplus \ldots \oplus K_{n}^{\prime}$ if either

(a) Warfield 72 End $N$ is a local ring or

(b) Arnold Lady 75 $N$ and $N^{\prime}$ have no quasi-summands in common.

In this case, $N^{\prime}$ is isomorphic to $K_{1}^{\prime} \oplus \ldots \oplus K_{n}^{\prime}$.

An indecomposable $M \in T F$ is purely indecomposable if $p$-rank $M=1$. In this case, End $M$ is a local ring, being a pure subring of $\mathbb{Z}^{*} \cong \operatorname{End}_{\mathbb{Z}^{*}} \mathbb{Z}^{*}$. Dually, $M$ is co-purely indecomposable if $M$ is indecomposable with rank $M=p$-rank $M+1$. There is a contravariant duality $F$ on $T F_{\mathbb{Q}}$ sending a purely indecomposable group $M$ to a co-purely indecomposable group $F(M)$ Arnold 72] (see Lady 77 for an alternate definition of the duality). Hence, $\mathbb{Q}$ End $F(M)$ is isomorphic to $\mathbb{Q}$ End $M$, a subring of the $p$-adic rationals $\mathbb{Q}^{*}$.

Following are some elementary properties of purely indecomposable and copurely indecomposable groups that are consequences of the definitions and the duality $F$.

Proposition 1 ( Arnold 72]). Let $M \in T F$.

(a) If $M$ is purely indecomposable, then:

(i) End $M$ is a pure subring of $Z^{*}$;

(ii) each pure subgroup of $M$ is strongly indecomposable;

(iii) if $K \in T F$ is a homomorphic image of $M$ with rank $K<\operatorname{rank} M$, then $K$ is divisible; and

(iv) two purely indecomposable groups $M$ and $N$ in $T F$ are isomorphic if and only if $\operatorname{rank} M=\operatorname{rank} N$ and $\operatorname{Hom}(M, N) \neq 0$; equivalently $M$ and $N$ are quasi-isomorphic.

(b) If $M$ is co-purely indecomposable, then:

(i) End $M$ is isomorphic to a subring of $\mathbb{Q}^{*}$, hence an integral domain;

(ii) each torsion-free homomorphic image of $M$ is strongly indecomposable;

(iii) if $K$ is a pure subgroup of $M$ with rank $K<\operatorname{rank} M$, then $M$ is a free $\mathbb{Z}_{(p)}$-module; and

(iv) two co-purely indecomposable groups $M$ and $N$ are quasi-isomorphic if and only if $\operatorname{rank} M=\operatorname{rank} N$ and $\operatorname{Hom}(M, N) \neq 0$.

(c) If $M$ is indecomposable with rank $\geq 2$ and $N$ is co-purely indecomposable with $\operatorname{rank} M<\operatorname{rank} N$, then $\operatorname{Hom}(M, N)=0$. 
(d) If $M$ is purely indecomposable with rank $\geq 3$ and $N$ is co-purely indecomposable with $\operatorname{rank} M=\operatorname{rank} N$, then $\operatorname{Hom}(M, N)=0$.

Remark 1 . There is a co-purely indecomposable $M \in T F$ with $p$-rank 3 and rank 4 that does not have either UDS property. In this case End $M$ has 3 maximal right ideals and $M$ is the summand of a non-Krull-Schmidt group of rank 12 Arnold 01. Remark]. In view of the following lemma, this group cannot be a summand of a non-Krull-Schmidt group of rank $8=2(\operatorname{rank} M)$. On the other hand, if $M \in T F$ and End $M$ has at least 4 maximal right ideals, then $M$ is a summand of a non-Krull Schmidt group of rank equal to $2(\operatorname{rank} M)$.

The next lemma is used in the proof of the main theorem. In view of Proposition 1(b)(i), the hypotheses are satisfied if $N$ is co-purely indecomposable.

Lemma 3. Assume that $N \in T F$ with End $N$ an integral domain and $M=N \oplus$ $N^{\prime}=K_{1} \oplus K_{2} \in T F$ with each $K_{i}$ indecomposable. If p-rank $N \leq 3$, then $N$ is isomorphic to some $K_{i}$.

Proof. The proof is a variation on a proof given in Arnold 01. Let $\pi$ be a projection of $M$ onto $N$ with kernel $N^{\prime}$, and $\pi_{i}$ a projection of $M$ onto $K_{i}$ for each $i$ with $1_{M}=\pi_{1}+\pi_{2}$. Then $1_{N}=\beta_{1}+\beta_{2}$, where $\beta_{i} \in$ End $N$ is the restriction of $\pi \pi_{i}$ to $N$ and $\beta_{i}(N)$ is contained in a subgroup $\pi\left(K_{i}\right)$ of $N$. Since End $N$ is an integral domain, $\mathbb{Q}$ End $N$ is a field and each $\beta_{i}$ is a unit in $\mathbb{Q E n d} N$.

For each $1 \leq i \leq 2$, let $I_{i}=\beta_{i}$ End $N$, a right ideal of End $N$. Then End $N=I_{1}+I_{2}$, since $1_{N}=\beta_{1}+\beta_{2}$. Each (End $\left.N\right) / I_{i}$ is bounded by a power of $p$ since $\beta_{i}$ is a unit in $\mathbb{Q}$ End $N$. Moreover, $I_{i} N$ is contained in $A_{i}=\pi\left(K_{i}\right)$ so that $\left[N: A_{i}\right]$ is finite. It now suffices to prove that $N \cong A_{i}$ for some $i$, in which case $N \cong K_{i}$.

If some $\left[N: A_{i}\right]=1$, then $N=A_{i}$ and the proof is complete. The next step is to assume that each $\left[N: A_{i}\right] \neq 1$ and reduce to the case that each $\left[N: A_{i}\right]=p$. Suppose, by way of induction, that $\left[N: A_{i}\right] \neq p$. Choose $x \in N \backslash A_{i}$ such that $p x \in A_{i}$. Then $A_{i} \subset A_{i}+\mathbb{Z} x$. If $N$ and $A_{i}+\mathbb{Z} x$ are not isomorphic, then replace $A_{i}$ by $A_{i}^{\prime}=A_{i}+\mathbb{Z} x$. If $N \cong A_{i}+\mathbb{Z} x$, say $f \in$ End $N$ with $f(N)=A_{i}+\mathbb{Z} x$, then replace $A_{i}$ by $A_{i}^{\prime}=f^{-1}\left(A_{i}\right)$. In either case, $\left[N: A_{i}^{\prime}\right]$ is a proper divisor of $\left[N: A_{i}\right]$.

The substitution of $A_{i}^{\prime}$ for $A_{i}$ doesn't change the hypothesis that End $N=I_{1}+I_{2}$ for right ideals $I_{i}$ of bounded index with $I_{i} N$ contained in $A_{i}$. In particular, $I_{i}^{\prime}=$ $f^{-1} I_{i}$ is an ideal of End $N$ (since $I_{i} N$ is a subgroup of $\left.f(N)\right), I_{i}^{\prime} N$ is contained in $A_{i}^{\prime}$, and $I_{i}^{\prime}+\Sigma\left\{I_{j}: j \neq i\right\}=$ End $N$ (since $f$ End $N=\Sigma f I_{i}$ is contained in $I_{1}+\Sigma\{f I j: j \neq i\}$ ). If $N \cong A_{i}^{\prime}$, then, by the construction of $A_{i}^{\prime}, N \cong A_{i}$. By induction, and the fact that $\left[N: A_{i}^{\prime}\right]$ is a proper divisor of $\left[N: A_{i}\right]$, the $A_{i}$ 's can be chosen with each $\left[N: A_{i}\right]=p$.

At this stage, End $N=I_{1}+I_{2}$ for right ideals $I_{i}$ of finite index in End $N$ with $I_{i} N$ contained in a subgroup $A_{i}$ of $N$ and $\left[N: A_{i}\right]=p$ for each $i$. Replace $I_{i}$ by $I_{i}+p$ End $N$, if necessary, to guarantee that $p$ End $N$ is contained in $I_{i}$ for each $i$. But $p$-rank $N \leq 3, p$ End $N \subseteq J$ End $N$, End $N$ is an integral domain, and $N /\left(M_{1} \cap \ldots \cap M_{n}\right) N \cong N / M_{1} N \oplus \ldots \oplus N / M_{n} N$ for maximal ideals $M_{i}$ of End $N$. Hence, End $N$ has at most 3 maximal right ideals $M_{1}, M_{2}$, and $M_{3}$ and $p$ End $N=M_{1}^{i_{1}} M_{2}^{i_{2}} M_{3}^{i_{3}}$ with $i_{1}+i_{2}+i_{3} \leq 3$. Furthermore, $p N \subseteq\left(I_{1} \cap I_{2}\right) N$, and $N /\left(I_{1} \cap I_{2}\right) N \cong N / I_{1} N \oplus N / I_{2} N$. After relabelling subscripts, if necessary, $I_{1}=M_{1}, N / I_{1} N=\mathbb{Z} / p \mathbb{Z}$, and $I_{1} N=A_{1}$. Finally, $I_{1}$ is principal by Nakayama's 
Lemma, since $p$ End $N \subseteq J$ End $N$ and $I_{1} / p$ End $N$ is principal. This shows that $A_{1}$ is isomorphic to $N$, as desired.

The point of the next lemma, as used in the proof of the main theorem, is that Lemma 2(a) applies to a group quasi-isomorphic to a direct sum of two purely indecomposable groups of the same rank.

Lemma 4. If $N \in T F$ is indecomposable and quasi-isomorphic to $A \oplus B$ for purely indecomposable groups $A$ and $B$ in $T F$ with rank $A=\operatorname{rank} B$, then $\operatorname{Hom}(A, B)=$ $0=\operatorname{Hom}(B, A)$ and End $N$ is a local ring.

Proof. Choose purely indecomposable pure subgroups $A$ and $B$ of $N$ and some least positive integer $i$ with $p^{i} N \subset A \oplus B \subset N$. Since $p$-rank $N=2, N / p^{i} N \cong$ $\mathbb{Z} / p^{i} \mathbb{Z} \oplus \mathbb{Z} / p^{j} \mathbb{Z}$ for some $1 \leq j \leq i$. Because $A$ and $B$ are purely indecomposable pure subgroups of $N, N /(A \oplus B) \cong \mathbb{Z} / p^{j} \mathbb{Z}$, say $N=A \oplus B+\mathbb{Z}(a, b)\left(1 / p^{j}\right)$ for some $a \in A \backslash p A$ and $b \in B \backslash p B$.

If $\operatorname{Hom}(A, B) \neq 0$ or $\operatorname{Hom}(B, A) \neq 0$, then $A$ and $B$ are isomorphic by Proposition 1(iv). Moreover, $C=N / A$ is purely indecomposable and quasi-isomorphic to $B$. Hence, $C \cong A \cong B$ and $\operatorname{Hom}(C, N) C=N$. By Baer's Lemma Arnold 82, $A$ is a summand of $N$, a contradiction to the assumption that $N$ is indecomposable.

Now assume that $\operatorname{Hom}(A, B)=0=\operatorname{Hom}(B, A)$. Then $A$ and $B$ are fully invariant subgroups of $N=A \oplus B+\mathbb{Z}(a, b)\left(1 / p^{j}\right)$. Thus, End $N$ is the pullback of a homomorphism $A \rightarrow \mathbb{Z} / p^{j} \mathbb{Z}$ with kernel $p^{j} A$ and a homomorphism $B \rightarrow \mathbb{Z} / p^{j} \mathbb{Z}$ with kernel $p^{j} B$. It follows that End $N / p^{j}$ End $N \cong \mathbb{Z} / p^{j} \mathbb{Z}$, whence End $N$ is a local ring.

\section{THE MAIN THEOREM}

Theorem 1. If $M \in T F$ and $\operatorname{rank} M \leq 9$, then $M$ is a Krull-Schmidt group.

Proof. Let $N$ be an indecomposable summand of $M$ of minimal rank and $M=N$ $\oplus N_{1} \oplus \ldots \oplus N_{m}=K_{1} \oplus \ldots \oplus K_{n}$ with each $N_{i}$ and $K_{j}$ indecomposable. Then $\operatorname{rank} N \leq 4, \operatorname{rank} N \leq \operatorname{rank} N_{j}$, and $\operatorname{rank} N \leq \operatorname{rank} K_{i}$ for each $i$ and $j$, since rank $M \leq 9$ and $N$ is an indecomposable summand of $M$ of minimal rank.

If $p$-rank $N \leq 1$, then End $N$ is a local ring, as noted above. In this case, by Lemma $2(\mathrm{a}), N_{1} \oplus \ldots \oplus N_{m}$ is isomorphic to $K_{1}^{\prime} \oplus \ldots \oplus K_{n}^{\prime}$ for subgroups $K_{i}^{\prime}$ of $K_{i}$. It follows, by an induction on the rank of $M$, that $M$ is a Krull-Schmidt group. In particular, if $p$-rank $N=\operatorname{rank} N$, then $N$ is free and cyclic, hence of $p$-rank 1 .

If $N$ and $N_{1} \oplus \ldots \oplus N_{m}$ have no quasi-summands in common, then, by Lemma 2(b), the proof is completed by an induction on the rank of $M$.

In view of the preceding remarks, it is now sufficient to assume that $M$ is reduced, $2 \leq p$-rank $N<\operatorname{rank} N \leq 4$ for each indecomposable summand $N$ of minimal rank, and if $M=N \oplus N^{\prime}$, then $N$ and $N^{\prime}=N_{1} \oplus \ldots \oplus N_{m}$ have a quasi-summand in common. Under these assumptions, $M$ has no rank-1 quasi-summands. This is because the only rank-1 groups in $T F$ are $\mathbb{Z}_{(p)}$ and $\mathbb{Q}$ and, since $M$ is reduced, any rank- 1 quasi-summand must actually be a summand isomorphic to $\mathbb{Z}_{(p)}$. The strategy of the remainder of the proof is to show that $N$ must be isomorphic to some $K_{i}$, in which case the cancellation property for $N \in T F$ and an induction on the rank of $M$ shows that $M$ is a Krull-Schmidt group.

First assume that rank $N=4, p$-rank $N=3$. Then $N$, being indecomposable, is co-purely indecomposable, hence strongly indecomposable by Proposition 1 . Thus, 
$N_{1} \oplus \ldots \oplus N_{m}$ is quasi-isomorphic to $N \oplus L$ for some $L$ of rank $\leq 1$. To see this, recall that $N$ and $N_{1} \oplus \ldots \oplus N_{m}$ have a quasi-summand in common, $N$ is strongly indecomposable, $\operatorname{rank} N \geq \operatorname{rank} N_{i}$, and rank $N+\sum_{i} \operatorname{rank} N_{i}=4+\sum_{i} \operatorname{rank} N_{i} \leq 9$. Since $M$ has no rank-1 quasi-summands, $L=0, m=1$, and $n=2$. But $T F_{\mathbb{Q}}$ is a Krull-Schmidt category so that $M=N \oplus N_{1}=K_{1} \oplus K_{2}$ has rank 8 with $N$ quasi-isomorphic to $N_{1}, K_{1}$, and $K_{2}$. By Lemma $3, N$ is isomorphic to either $K_{1}$ or $K_{2}$, as desired.

Next, consider the case that rank $N=4$ and $p$-rank $N=2$. If $N$ is strongly indecomposable, then, as above, $M=N \oplus N_{1}=K_{1} \oplus K_{2}$ has rank 8 and $N$ is quasi-isomorphic to $N_{1}, K_{1}$, and $K_{2}$. By Example 1, $N$ has the UDS property so that $N$ is isomorphic to either $K_{1}$ or $K_{2}$, as desired. If $N$ is not strongly indecomposable, then $N$ is quasi-isomorphic to $A \oplus B$, where $A$ and $B$ are purely indecomposable groups with $p$-rank 1 and rank 2 . This is because $M$ has no rank-1 quasi-summands. Now apply Lemmas 2 and 4 and induction on the rank of $M$ to see that $M$ is a Krull-Schmidt group.

The only remaining case is that $p$-rank $N=2, \operatorname{rank} N=3$. In this case $N$ is co-purely indecomposable, hence strongly indecomposable by Proposition 1 . Since $N$ and $N_{1}$ have a quasi-summand in common, $N_{1}$ is quasi-isomorphic to $N \oplus A$ for some pure subgroup $A$ of $N_{1}$ with $1 \leq p$-rank $A<\operatorname{rank} A \leq 3$. This is because $M$ has no rank-1 quasi-summands and rank $M \leq 9$.

If $A$ has $p$-rank 1, then $\operatorname{Hom}(A, N)=0$ by Proposition 1(c) and (d), since $A$ is purely indecomposable with $2 \leq \operatorname{rank} A \leq 3=\operatorname{rank} N$, and $N$ is co-purely indecomposable. In this case, $\operatorname{Hom}(A, M)=\operatorname{Hom}(A, A)$. It follows that $A$ is a pure fully invariant subgroup, hence equal to a subgroup of some $K_{i}$, say $K_{1}$. Thus, $N \oplus\left(N_{1} / A\right)$ is isomorphic to $\left(K_{1} / A\right) \oplus K_{2} \oplus K_{3}$ and induction on the rank of $M$ completes the proof.

Finally, assume that $A$ has $p$-rank 2. Then $\operatorname{rank} A=3=\operatorname{rank} N$ and $A$ and $N$ are both co-purely indecomposable. If $\operatorname{Hom}(A, N)=0$, then, as above, $M$ is a Krull-Schmidt group. Finally, if $\operatorname{Hom}(A, N) \neq 0$, then $A$ is quasi-isomorphic to $N$, since $A$ and $N$ are both co-purely indecomposable modules with the same rank Hence, $M=N \oplus N_{1}=K_{1} \oplus K_{2} \oplus \ldots \oplus K_{n}$ has rank 9 with $n \leq 3$. If $n=3$, then $N$ is quasi-isomorphic to $K_{1}, K_{2}$ and $K_{3}$ by the minimality of the rank of $N$. In this case, Example 1 yields $N$ isomorphic to some $K_{i}$. If $n=2$, then, by Lemma $3, N$ is isomorphic to some $K_{i}$, as desired.

Example 2 (Arnold 01]). There is a rank-10 group in TF that is not a KrullSchmidt group.

Proof. The argument is briefly outlined. There is $M \in T F$ of $p$-rank 4 and rank 5 such that $M \cong$ End $M$, a subring of an algebraic number field with exactly four maximal ideals $M_{1}, M_{2}, M_{3}$, and $M_{4}$, and $p M=p$ End $M=M_{1} \cap M_{2} \cap M_{3} \cap M_{4}$. Furthermore, there are subgroups $A_{1}$ and $A_{2}$ of $M$ not isomorphic to $M$ with $\left(M_{1} \cap M_{2}\right) M \subset A_{1}$ and $\left(M_{3} \cap M_{4}\right) M \subset A_{2}$. It follows that there is $B \in T F$ with $M \oplus B=A_{1} \oplus A_{2}$, a rank 10 group in $T F$ that is not a Krull-Schmidt group.

\section{REFERENCES}

[Arnold 72] Arnold, D. A duality for torsion-free modules of finite rank over a discrete valuation ring, Proc. Lond. Math. Soc. (3) 24 (1972), 204-216.

[Arnold 82] Arnold, D. Finite Rank Torsion-Free Abelian Groups and Rings, Lect. Notes in Math. 931, Springer-Verlag, New York, 1982. MR 84d:20002 
[Arnold 00] Arnold, D. Abelian Groups and Representations of Finite Partially Ordered Sets, CMS Books in Mathematics, Springer-Verlag, New York, 2000. MR 2001g:16030

[Arnold 01] Arnold, D. Direct sum decompositions of torsion-free abelian groups of finite rank, Abelian Groups, Rings, and Modules (Proc. of 2000 Perth Conf.), Cont. Math., AMS, Providence, Rhode Island, 2001, 65-74. CMP 2001:09

[Arnold Dugas 00] Arnold, D. and Dugas, M. Co-purely indecomposable modules over a discrete valuation ring, J. Pure and Appl. Alg. 161 (2001), 1-12.

[Arnold Lady 75] Arnold, D. and Lady, E.L. Endomorphism rings and direct sums of torsionfree abelian groups, Trans. Amer. Math. Soc. 211 (1975), 225-237. MR 54:5370

[Fuchs 73] Fuchs, L. Infinite Abelian Groups, Vol. II, Academic Press, New York, 1973. MR 50:2362

[Goldsmith May 99] Goldsmith, B. and May, W. The Krull-Schmidt problem for modules over valuation domains, J. Pure and Appl. Alg. 140 (1999), 57-63. MR 2000d:13016

[Lady 75] Lady, E.L. Nearly isomorphic torsion-free abelian groups, J. Alg. 35 (1975), 235-238. MR 51:5801

[Lady 77] Lady, E.L. Splitting fields for torsion-free modules over discrete valuation rings, J. Alg. 49 (1977), 261-275. MR 58:22039

[Walker 64] Walker, E.A. Quotient categories and quasi-isomorphisms of abelian groups, Proc. Colloq. Abelian Groups, Budapest, 1964, 147-162. MR 31:2327

[Warfield 72] Warfield, R.B. Jr. Exchange rings and decompositions of modules, Math. Ann. 199 (1972), 31-36. MR 48:11218

Department of Mathematics, Baylor University, Waco, Texas 76798-7328

E-mail address: David_Arnold@baylor.edu 